\title{
To Study the Characteristics of Low Base Station Antenna in Respect of Propagation Loss
}

\author{
Ravi Prakash Sharma ${ }^{1}$, Santosh Tripathi ${ }^{2}$, Anil Kumar $^{3}$, A. K. Jaiswal ${ }^{4}$ \\ Shiats, Allahabad, India
}

\begin{abstract}
This paper proposes a study of the characteristics of low base station antenna on urban/sub urban road. We have to investigate the effect of various parameters as frequency, low base station antenna height, distance between low base station antenna and subscriber station and develop a mathematical approach. Our analysis will be useful to develop a link budget on the basis of propagation loss characterization for transmission to allow adequate reception.
\end{abstract}

Keywords: propagation loss, antenna height, frequency, low base station antenna, reflection, diffraction

\section{Introduction}

The environment between the transmitter and receiver in a wireless communication system has a significant effect on the performance and to maintain quality of service of the system. Propagation loss is an important element which must be kept within predefined range in order to get expected performance of the system. In this paper, we clarify propagation characteristics of a low base station antenna on the road to measure propagation loss. They show the characteristics of the basic propagation loss such as the distance and frequency dependence, effect of base station and subscriber's antenna heights. Furthermore, on the basis of these results, the service area is predicted for a given ranges of various parameters considering both free space propagation loss as well as the loss due to different transmission mechanism like reflection, diffraction and scattering in Non-Line of sight environment.

\section{Method}

Propagation loss model can be classified under two categories: statistical type and deterministic type. The statistical method is based on they are measured and averaged losses along typical classes of radio link. The deterministic methods are based on the physical laws of wave propagation. They are expected to produce more accurate and reliable predictions on the propagation loss model than the statistical method. The practical scenario of the arrangement of buildings in an urban or sub urban area is not so simple under a generalized propagation loss. Therefore, the propagation loss model that considers the more structural varieties will give better perfection in design. The Walfisch-Bertoni model is semi-deterministic model valid for situations of uniform building heights and spacing in urban and sub-urban area. The Walfisch-Bertoni model considered total propagation loss consists of three elements: free space loss, diffracting loss from rooftop to street and loss due to multiple screen diffraction.

However Propagation loss is direct function of frequency of wireless communication system, higher frequency is associated with more path loss shown in Fig.1, hence with higher frequency a signal can travel a shorter path than that of lower frequency, where $\mathrm{c}$ is speed of light, $f$ transmitting frequency of signal, $d$ separation between low base station antenna and subscriber's antenna. Let overall propagation loss for subscriber station at a distance $d$ from the low base station antenna of height $H$ be $P_{d}$ [3].

$$
\begin{gathered}
\text { Free space loss }=-10 \log _{10}\left[\frac{c}{4 \pi f d}\right]^{2} \\
P_{d}=80-18 \log _{10}(H)+40\left(1-\frac{4 H}{1000}\right) \log _{10}(d)+21 \log _{10}(f)
\end{gathered}
$$

From these equations it is clear that free space propagation loss only depends on two factors separation between base station antenna and transmitting frequency and overall propagation loss depends on different transmission mechanism like reflection, diffraction and scattering in Non-Line of sight environment, this model is valid for a range of $\mathrm{H}$ from 0 to $50 \mathrm{~m}$.

It is clear from Fig.3 Frequency dependence become almost insignificant; the overall propagation loss is smaller for high frequency because for high frequency the base mobile propagation path tends to become a line of sight path. The Fresnel zone radius decreases as the square root of frequency. There are two types of propagation loss for frequency. One is free space loss and the other is based on the Fresnel-zone radius and road width. When road width is more than twice the first Fresnel-zone radius, loss is almost the same as free space loss. When road width is less than the radius, loss becomes larger than free space loss [1]. 
Dependence on base station antenna height: Fig.5 shows when the low base station antenna height increases the overall loss decreases because of the probability of line of sight. Propagation loss is almost independent of base station antenna height at free space condition because propagation path is along the line of sight, without obstacles such as cars. However, loss does depend on height, when there are cars on the road. It is clear from Fig. 2 and Fig.4 when subscriber is moving away (toward base station) from low base station antenna $(\mathrm{H}=10 \mathrm{~m})$ then losses increases rapidly. Overall Loss is calculated under the same conditions except that antenna height now varies. Overall loss decreases because diffraction loss is reduced as base station antenna height becomes higher, as shown in Fig.5. Reflection waves become dominant because of diffraction loss increase, as mobile station is near obstacle.

\section{Result and Discussion}

Our above analyzed results provide better and simpler solution to calculate propagation losses under various transmitting conditions:

(i) When there are no obstacles on the straight road on which the base station is located, the distance over which a radio wave propagates with only free-space loss increases with frequency and separation between low base station antenna and subscriber antenna.

(ii) If separation increases between low base station antenna and subscriber antenna we must consider extra path loss regardless of transmission frequency.

(iii) The additional propagation loss due to increase in transmission frequency and increase in separation between the low base station antenna and subscriber antenna can be minimized by increasing the low base station antenna height.

\section{Conclusion}

In this paper, we have considered the three parameters i.e. low base station antenna height, transmitting frequency and separation between base station antenna and subscribers antenna those have a good chance to while designing and upgrading wireless communication system. The parameters in Walfisch-Bertoni path loss model based on ITU M.1225 Recommendation along with city structure may also affect in propagation loss when above three considered parameters are under change.

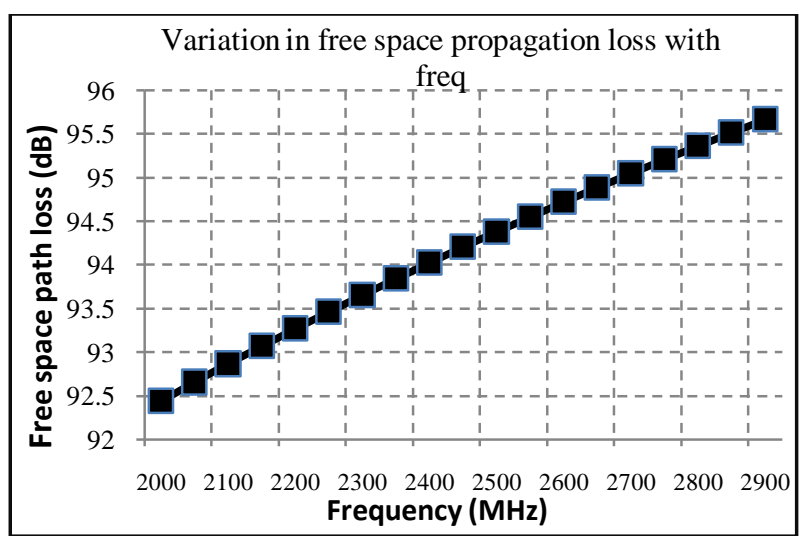

Fig.1

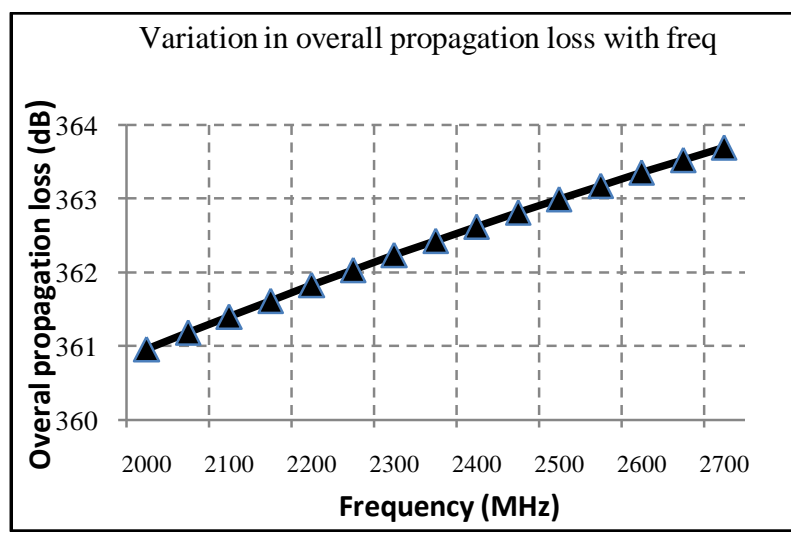

Fig.3

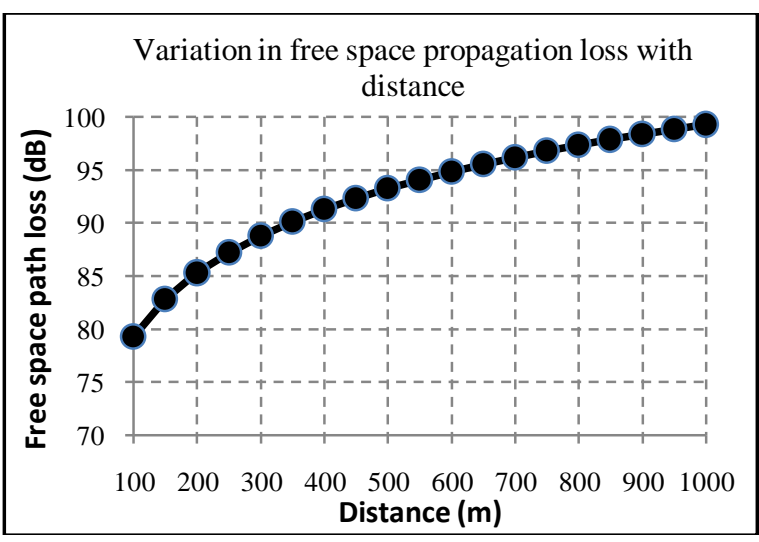

Fig. 2

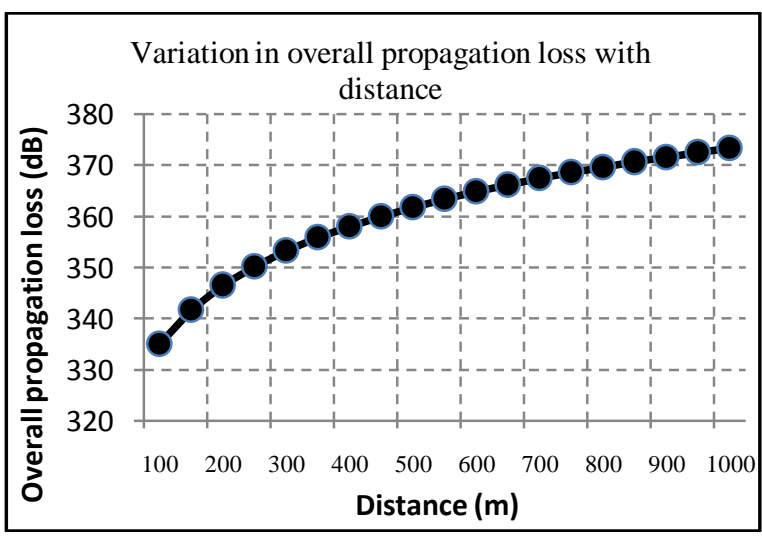

Fig.4 


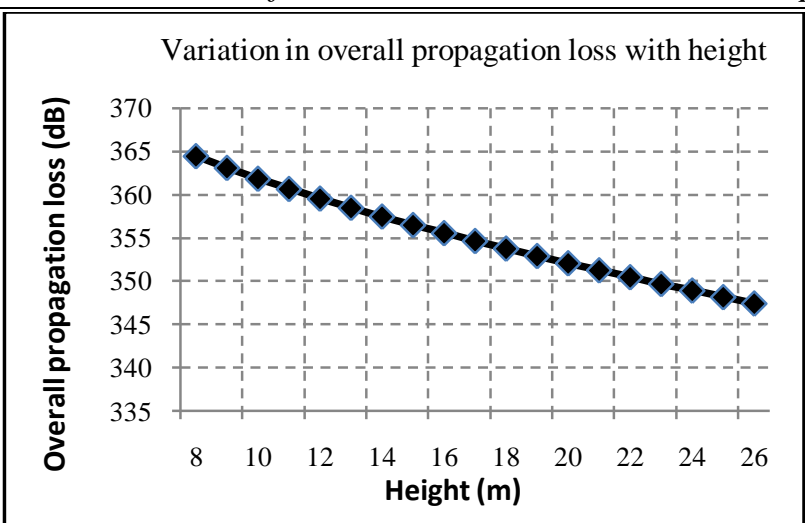

Fig.5

\section{References}

[1] S. Kozono and A. Taguchi, "Propagation characteristics of low base-station antenna on urban road," in IEEE AP-SIURSI, vol. 111,pp. 1250-1253, Syracuse, NY, 1988.

[2] Walfisch, J \& Bertoni, H, L. (1988) "A theoretical model of UHF propagation in urban environments", IEEE Transaction on Antennas and Propagation. Vol 36. No 12. Pp. 1788-1796.

[3] KurratulAin, M. M. Rahman Tarafder, 'Path loss compensation technique for Wimax Technology based communication system', in IJEST, vol.3 No.9 September 2011.

[4] J. Walfisch and H.L. Bertoni, '’A Theoretical Model of UHF Propagation in Urban Environments'” IEEE trans. Ant. Prop, vol. AP36, pp. 1788-1796, Dec 1988.

[5] R. J. C. Bultitude and G. K. Bedal, "Propagation characteristics on microcellular urban mobile radio channel at $910 \mathrm{MHz}$," IEEE J. Selected Areas Commun. vol. 7, pp. 31-39, Jan. 1989

[6] A. J. Rustako, et al., "Attenuation and diffraction effects from truck blockage of an 11-GHz line-of-sight microcellular mobile radio path,” IEEE Trans. Veh. Technol., vol. 40, pp. 211-215, Feb. 1991. 